\author{
Military Technical College \\ Kobry El-Kobbah, \\ Cairo, Egypt.
}

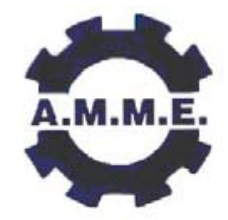

$13^{\text {th }}$ International Conference on Applied Mechanics and Mechanical Engineering.

\title{
THEORETICAL AND EXPERIMENTAL INVESTIGATION OF THE ANALYSIS OF CHATTER VIBRATION AND STABLITY FOR ORTHOGONAL CUTTING IN TURNING
}

\author{
TURKES* E. and ORAK** S.
}

\begin{abstract}
Increasing of the Maximum Metal Removal Rate (MMRR) in machine tools requires high width of metal removing and machining speeds. This condition cause to change cutting speed and arises vibration on cutting tool. This phenomenon leads to such problems as detrimental effects on the machined surface finish and decreasing the machining efficiency and has adverse effect on the work surface finish, high machining force, tool wear and tool life. Due to the dynamic interactions between cutting tool and workpiece, there occurs self-excited chatter vibration. In this study, chatter prediction is investigated for orthogonal cutting in turning operations. Therefore, the linear analysis of the single degree of freedom (SDOF) model is performed by applying oriented transfer function (OTF) and $\tau$ decomposition form to Nyquist criteria. Chatter frequency predictions obtained from both forms were compared with modal analysis and cutting tests. Computer programs required for both forms were written by MATLAB program.
\end{abstract}

\section{KEY WORDS}

Orthogonal cutting, Chatter frequency, linear analysis.

\footnotetext{
Research Assist., Dpt. of Mech. Eng., Dumlupınar University, Turkey.

** Associate professor, Dpt. of Mech. Eng., Eskişehir Osmangazi University, Turkey.
} 


\section{INTRODUCTION}

There are two types of vibrations in metal removal, generally. These are forced and self-excited vibrations. Forced vibrations occur as a result of the total effects of the mechanical movements of the machine tool. Or these vibrations are carried out if there is an external, periodic force acting on the cutting system. On the other hand, selfexcited vibrations develop due to a built in mechanism in the cutting system. Chatter is a self-excited type of vibration that occurs in metal cutting if the chip width is too large with respect to dynamic stiffness of the cutting system. Under the such as conditions these vibrations start and quickly grow. During cutting the cutting force becomes periodically variable, reaching considerable amplitudes and the machined surface becomes undulated. Also, change in cutting speed originates from the change in the shear angle. Chatter vibration leads to such production-limiting effects as reduce in dimensional accuracy in workpieces, poor surface finish, wear and breakage of cutting tools, damage to machine tools.

There are two main sources of self-excitation in metal cutting. These are mode coupling and regeneration of waviness on machined surface [1,2]. The quality of the surface being machined is of great importance especially in defense, space and moulding industries, and in cutting metals such as aluminum that are soft and easiest to cut [3-6].

Researchers, today, brought forward analysis and control techniques through various models for the prediction of chatter vibration. But since the mechanics and dynamics of cutting could not be put forward satisfactorily, a complicated model that is capable of expressing metal removal dynamics properly still does not exist [7]. Analysis of chatter vibrations are realized by the process of linear and nonlinear forces. All chatter analysis techniques begin with a model of machining force process and a model of the toolworkpiece structure. Machining operation is dealt with in a closed-loop form. To prevent chatter vibration, Stability Lobe Diagrams (SLDs) are plotted from harmonic solution of the characteristic equations of the system in determining the critical speed and cutting depths. Two models exist to do this. These are Nyquist criterion and Time Domain Simulation (TDS) techniques.

Analytical and numerical analysis of the cutting process can be made using these techniques. Some simplifications are made in analytical analysis. These are the acceptance that cutting force affects the surface of the workpiece at a constant angle and is linearly proportioned to the area of chip removed, and rigidity and damping of the machine tool are linear. Numerical models, on the other hand, can eliminate some deficiencies of analytical system by an approach of the real system [7-13]. Nyquist criterion is applied so that cutting conditions should be stable. This procedure is continued until the critical cutting depth, which provides stable cutting requirement, is determined. In TDS technique, the closed-loop dynamical model of the machining operation is simulated for a particular set of cutting conditions.

Steady state tool/workpiece displacement and machining force signals are examined to determine system stability. Displacement of the tool according to the workpiece and signals of cutting force is investigated for the determination of system stability. Thus effort is given to determine the critical depth of cut which provides the best stability. One of the most important superiority of TDS is that it covers nonlinear characteristics concerned in chatter analysis. Its disadvantage, however, is that simulations involve numerous runs that make the calculation difficult, and even there may be excessive 
loss of time. Due to these disadvantages, efforts to develop analytical methods in plotting SLD are still made by researchers, and new analytical methods have been devised [14-17].

In this study chatter vibration was predicted in orthogonal cutting with SDOF turning system by two analytical forms. Firstly, this cutting process modeled according to OTF and $\tau$ decomposition forms. Then, in the 3rd section of this study stability of this system was investigated by applying oriented transfer function (OTF) and $\tau$ decomposition form to Nyquist criteria. Finally, results obtained from both forms were compared with the modal analysis conducted and the results of cutting test.

\section{MODELLING OF THE CUTTING SYSTEM}

Chatter prediction and stability analysis was conducted analytically for a turning process with SDOF in such a way that OTF and $\tau$ decomposition form was applied to Nyquist criterion comparatively. Since the dynamic cutting operations are very complicated, turning system to be investigated in this study was modeled by massspring-damper as is seen in Figure 1.

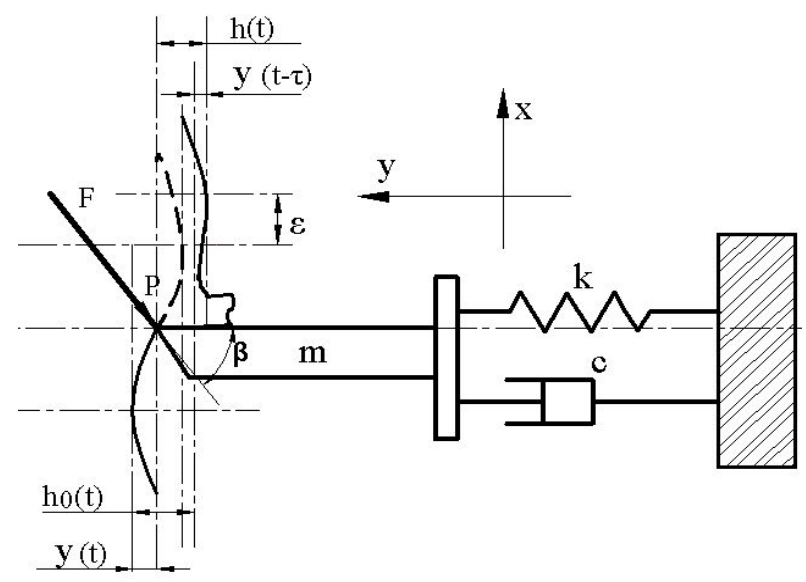

Fig. 1. Modeling of SDOF system in turning.

The equation of motion of the cutting system in the feed direction (y) was formed as follows,

$m_{y} \cdot \ddot{y}(t)+c_{y} \cdot \dot{y}(t)+k_{y} \cdot y(t)=F_{y}(t)$

where $\mathrm{F}_{\mathrm{y}}(\mathrm{t}), F_{y}(t)=-F(t) \cdot \cos \beta$ can be written in terms of the cutting force and this is;

$F_{y}(t)=w \cdot C \cdot h(t)$

can be expressed by $(w)$, chip width $(\mathrm{mm})$; C, specific cutting energy of the material $\left(\mathrm{N} / \mathrm{m}^{2}\right)$ and $\mathrm{h}(\mathrm{t})$, instantaneous chip thickness. Instantaneous chip thickness, however; 
$h(t)=h_{0}-y(t)+y(t-\tau)$

can be written clearly according to the geometry in Figure 1 . Where $[y(t)-y(t-\tau)]$ is the displacement of end point $\mathrm{P}$ of the tool on the $\mathrm{y}$ axis and is called as dynamic chip load or dynamic chip width. With the help of these equations, chatter prediction of the cutting system was investigated analytically by OTF and t decomposition respectively.

\section{Oriented Transfer Function}

Oriented transfer function of the single degree of freedom system in Figure 1 is obtained from equations (1), (2) and (3) [1]

$m_{y} . \ddot{y}(t)+c_{y} \cdot \dot{y}(t)+k_{y} \cdot y(t)=F_{y}(t)=w \cdot C \cdot h(t)$

If equation of motion is written in Laplace domain, in order for the OTF between chip depth of the system $y(t)$, which is being removed now, and the force in the feed direction to be written,

$m_{y} \cdot s^{2} \cdot y(s)+c_{y} \cdot s \cdot y(s)+k_{y} \cdot y(s)=F_{y}(s)$

where, as seen in Figure 2, transfer function of the open-loop between $y(s)$ and $F_{y}(s)$, can be written as folows,

$G(s)=\frac{y(s)}{F_{y}(s)}=\frac{1}{m \cdot s^{2}+c . s+k}$

on the other hand, closed-loop transfer function between dynamic and reference chip loads is obtained by taking Laplace transformation of the equation (3) and substitution of $y(s)=G(s) \cdot F_{y}(s)$ into $h(s)$;

$$
\frac{h(s)}{h_{0}(s)}=\frac{1}{1+C \cdot w \cdot G(s) \cdot\left(1-e^{-\tau s}\right)}
$$

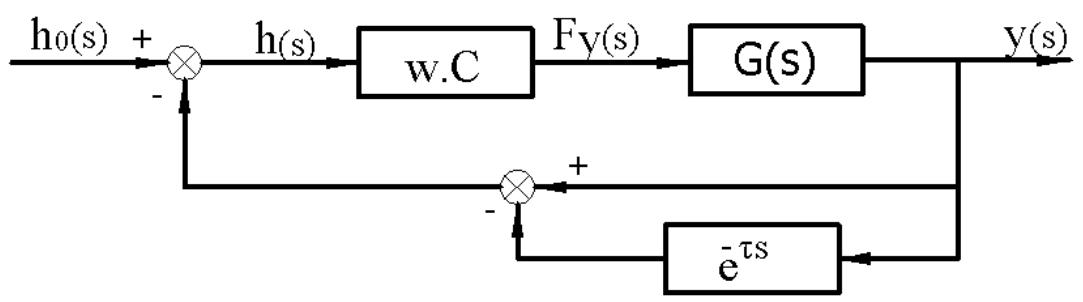

Fig. 2. Block diagram of chip depth for the turning system with SDOF.

Closed-loop transfer function between $y(s)$ and $h_{0}(s)$ is written as the equation (4) 
$m_{y} \cdot \ddot{y}(t)+c_{y} \cdot \dot{y}(t)+k_{y} \cdot y(t)=w \cdot C \cdot\left[h_{0}-y(t)+y(t-\tau)\right]$

and obtained by applying Laplace transformation on both sides.

$$
\frac{y(s)}{h_{0}(s)}=\frac{C \cdot w \cdot G(s)}{1+C \cdot w \cdot G(s) \cdot\left(1-e^{-\tau s}\right)}
$$

Denominators of the equations (7) and (9) are the characteristic equation of the system.

\section{$\tau$-Decomposition Form}

Taking the cutting force, which changes in the course of time, proportionally with the surface area of the chip removed from the surface is a general acceptance of linear modeling. For this reason, the constant component of the cutting force is neglected but variable component produced by dynamic chip load is taken into account. According to this calculation, the variable force can be written as,

$$
F_{v}(t)=C \cdot w \cdot[y(t)-y(t-\tau)]
$$

This is shown in the block diagram in Figure 3

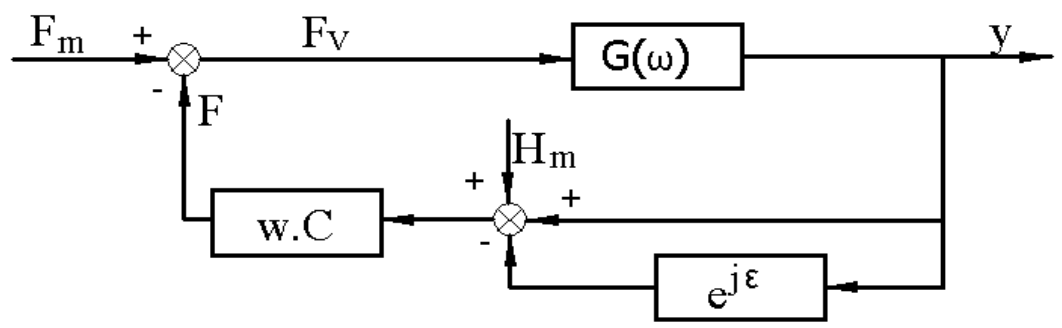

Fig. 3. Block diagram of cutting forces for the turning system with SDOF.

In the diagram, $F_{m}$ is the average cutting force, $H_{m}$ is average chip load. Where, equation (1) can be write as:

$m_{y} \cdot \ddot{y}(t)+c_{y} \cdot \dot{y}(t)+k_{y} \cdot y(t)=-\mathrm{F}(\mathrm{t}) \cdot \cos \beta$

If the equation for $\mathrm{T}$ decomposition form is written independent of time and according to the course taken by the relative movement of tool tip to the workpiece,

$l=V \cdot t+x$

where, $V$ is the linear speed in the rotation direction of the workpiece $(\mathrm{m} / \mathrm{s}), x$ is the displacement of tool tip independently from workpiece on the axis $x$ during cutting $(\mathrm{m})$. When derivative of the equation (10) is taken as time related,

$$
\frac{d l}{d t}=V+\dot{x} \approx V
$$


where, $(\dot{x})$ was neglected, since, according to linear speed, it would have a small value for linear modeling. With these simplifications in equation (11),

$$
\begin{aligned}
& \dot{y}(t)=\frac{d y}{d t}=\frac{d y}{d l} \cdot \frac{d l}{d t}=(V+\dot{x}) \cdot \frac{d y}{d l} \approx V \cdot \frac{d y}{d l} \rightarrow y^{\prime \prime}=\frac{d y}{d l} \\
& \ddot{y}(t)=\frac{d^{2} y}{d t^{2}}=\frac{d}{d t} \cdot\left[(V+\dot{x}) \cdot \frac{d y}{d l}=\ddot{x} \cdot\right] \frac{d y}{d l}+(V+\dot{x}) \cdot \frac{d^{2} y}{d l^{2}} \cdot \frac{d l}{d t} \approx V^{2} \cdot \frac{d^{2} y}{d l^{2}} \rightarrow y^{\prime \prime}=\frac{d^{2} y}{d l^{2}}
\end{aligned}
$$

the following equation is obtained,

$$
m \cdot V^{2} \cdot y^{\prime \prime}+c \cdot V \cdot y^{\prime}+k \cdot y=-w \cdot C \cdot[y(l)-y(l-\pi d l)] \cdot \cos \beta
$$

thus, according to constant $(l)$ equation of motion is obtained instead of time dependent one, $\mathrm{T}$. If both sides of the equation (14) is reduced for simplification,

$$
c_{1}=\frac{c}{m \cdot V} \quad k_{1}=\frac{k}{m \cdot V^{2}} \quad F_{1}=\frac{-w \cdot C \cdot \cos \beta}{m \cdot V^{2}}
$$

and if these values placed and the equation is equal to zero, and Laplace transformation is applied, characteristic equation of the system is obtained,

$$
s^{2}+c_{1} \cdot s+\left(k_{1}-F_{1}\right)+F_{1} \cdot e^{-s d \pi}=0
$$

with further reducing,

$$
a_{2}=1 / F_{1} \quad a_{1}=c_{1} / F_{1} \quad a_{0}=\left(k_{1}-F_{1}\right) / F_{1}
$$

is obtained.

$$
e^{s d \pi}=\frac{-1}{a_{2} \cdot s^{2}+a_{1} \cdot s+a_{0}}
$$

According to Nyquist criterion, right side of this equation expresses Nyquist plane curve $U_{2}(s)$ and the left side expresses critical orbit $U_{1}(s)$. If $\mathrm{s}=\mathrm{j} \omega$ is taken in the equation, roots of the characteristic equation (16) is found by equalizing the magnitude of right side of the equation to 1 ,

$$
\begin{aligned}
& \frac{1}{\left|\left(-a_{2} \cdot \omega^{2}+a_{0}\right)+j \cdot a_{1} \cdot \omega\right|}=1 \\
& \left(-a_{2} \cdot \omega^{2}+a_{0}\right)^{2}+\left(a_{1} \cdot \omega\right)^{2}=1 \\
& a_{2}^{2} \cdot \omega^{4}+\left(-2 \cdot a_{2} \cdot a_{0}+a_{1}^{2}\right) \cdot \omega^{2}+a_{0}^{2}-1=0
\end{aligned}
$$

thus positive real root of this equation will give the chatter frequency of the system. 


\section{INVESTIGATING THE STABILITY OF THE CUTTING SYSTEM}

Two methods are used for the determination of stable areas for chatter-free spindle speed or the system of spindle/tool holder/cutting tool. The first method is the determination of the natural frequency of the system and mode shapes by measuring transfer functions by using an impact hammer and accelerometer. Analytical predictions of performance are then made from this information. The second method is perform of cutting tests. This method gives the cutting ability of spindle/cutting tool in a better completeness, but requires a number of tests to be performed. One of the first developed analysis techniques was established by Meritt (1965). This technique is based on the investigation of stability and plotting the SLD from the solution of the characteristic equation of the system depending on the critical parameters such as axial cutting depth of the system and spindle speed. Two chatter analysis techniques are used in plotting SLDs. The former is Nyqist technique, which has been used by many researchers so far. According to this technique, stability of the technique is investigated in accordance with cutting conditions that are taken as basis (i.e. depth of cut and spindle speed) by constructing the characteristic equation of the system, and the procedure is repeated until the critical value which provides stability is determined.

The second technique is the TDS technique, in which cutting conditions of the cutting operation of the closed-loop dynamic model and are stimulated for a chosen group. In this technique, tool/workpiece displacement which continually change and signals of machining force are examined until marginal stability is obtained according to a chosen critical parameter (i.e. cutting depth). Since this technique involves most outstanding aspects like nonlinear characteristics of cutting operation, it is a more effective technique of analysis. But it also has the disadvantages mentioned before. Also, in studies conducted so far in the field of SLD drawings, at least one difference has been observed between analytic predictions and TDS techniques [1,12]. Analytic prediction is realized by iterative analytical solution of time-changing force coefficient of mathematical model formed by applying it to the distribution of Fourier series. This analysis is made with the acceptance that the force process is linear according to feed and depth and that it doesn't depend clearly on cutting speed. In addition to this, studies have been made recently to mount on the machine tool a sensor/actuator on the tool/tool holder system designed to suppress chatter vibration $[13,18,19]$. In the cutting process dealt with in this section, SLDs, which give stable and unstable cutting regions for chatter-free cutting, will be drawn according to two different forms explained in the previous section depending cutting depth and spindle speed.

\section{According to Oriented Transfer Function}

If the denominator of the equation (7), which is the characteristic equation of the system, is equaled to zero,

$$
1+C \cdot w_{\lim } \cdot G(s) \cdot\left(1-e^{-\tau s}\right)=0
$$

where, $w_{\text {lim }}$, means chatter-free maximum cutting depth. The roots of this characteristic equation will give the chatter frequency of the system in the form of $s=\sigma+j \omega$. When the real part of the roots is zero $(s=j \omega)$, the system is critically stable and workpiece oscillates with constant vibration amplitude at chatter frequency $(\omega)$. If $s=j \omega$ is placed 
in characteristic equation for critical borderline stability analysis, equation (18), can be written as,

$1+C \cdot w_{\lim } \cdot G(j \omega) \cdot\left(1-e^{-\tau j \omega}\right)=0$

when it is placed in the equation (19) in the form of real and imaginary parts $G(j \omega)=\mathrm{Re}+j \mathrm{Im}$, characteristic equation can be written in the form of real and imaginary parts,

$\left\{1+C \cdot w_{\lim }[\operatorname{Re}(1-\cos \omega \tau)-\operatorname{Im} \sin \omega \tau]\right\}+j\left\{C \cdot w_{\lim }[\operatorname{Re} \sin \omega \tau+\operatorname{Im}(1-\cos \omega \tau)]\right\}=0$

For stability, both real and imaginary parts of the equation must be zero. If the imaginary part is equaled to zero first, $\operatorname{Re} \sin \omega \tau+\operatorname{Im}(1-\cos \omega \tau)=0$, the ratio of real and imaginary parts gives the phase angle $(\psi)$ of the root on Nyquist diagram,

$\tan \psi=\frac{\operatorname{Im}(\omega)}{\operatorname{Re}(\omega)}=\frac{\sin \omega \tau}{\cos \omega \tau-1}$

this value is the phase delay of the frequency transfer function of the system. If the halfangle formula in trigonometry is applied [1],

$\omega \tau=3 \pi+2 \psi$

is obtained. With this in mind, the phase effect in regenerative chatter vibration can be written as follows,

$n+\frac{\varepsilon}{2 \pi}=\frac{f}{\Omega}=f . \tau$

where, $f$ is frequency of the cutting tool $(\mathrm{Hz}) ; \Omega$ is the spindle speed $(1 / \mathrm{s}) ; \varepsilon / 2 \pi$ the fractional number of waves formed on he surface. It can be seen here that there is a phase shift between inner and outer waves $\varepsilon=3 \pi+2 \psi$. The corresponding spindle period and maximum spindle speed is found to be,

$$
\tau=\frac{2 n \pi+\varepsilon}{2 \pi f} \rightarrow \quad N=\frac{60}{\tau} \quad \mathrm{n}=0,1,2,3, \ldots
$$

The critical axial depth of cut can be found by equating the reel part of the characteristic equation to zero. Hence,

$w_{\text {lim }}=\frac{-1}{C \cdot \operatorname{Re}[(1-\cos \omega \tau)-(\operatorname{Im} / \operatorname{Re}) \sin \omega \tau]}, \frac{\operatorname{Im}(\omega)}{\operatorname{Re}(\omega)}=\frac{\sin \omega \tau}{\cos \omega \tau-1}, w_{\text {lim }}=\frac{-1}{2 \cdot C \cdot \operatorname{Re}(\omega)}$

is calculated. 


\section{According to $\tau$-Decomposition Form}

According to T-decomposition form, the characteristic equation of the system is equation (15). The roots of this equation are obtained from the solution of equation (17). Each positive real root $\left(\omega_{i}(j \omega)\right)$ is substituted back into the right side of the equation (16) to find $U_{2}\left(j \omega_{i}\right)$. The phase angle of the resulting number is computed as follows;

$$
\psi_{i}=\tan ^{-1} \frac{\operatorname{Im}\left(U_{2}\left(j \omega_{i}\right)\right)}{\operatorname{Re}\left(U_{2}\left(j \omega_{i}\right)\right)}
$$

Again, according to equation (22), value of time delay is found,

$$
\tau_{i}=\frac{\psi_{i}+2 \pi n}{\omega_{i}} \quad \mathrm{n}=0,1,2,3, \ldots
$$

Depending on this, maximum spindle speed to be gained in stable cutting is found as it is in equation (23). Finding critical axial depth of cut required for the borders of stable cutting, if organized and dealt with the way it is seen in equation (11) in Figure 3 and according to Nyquist criterion, can be found in relation to point $(-1,0 \mathrm{j})$ of the unit circle.

$$
\text { w.C.G } G(s) \cdot\left(1-e^{-\tau s}\right)=(-1,0 j)
$$

If the equation is reorganized in a form $s=j \omega$, it can be calculated by the following, expression

$$
w \cdot C \cdot G(\omega) \cdot\left(1-e^{-j \omega \tau}\right)=-1
$$

thus the borders of stable cutting corresponding to spindle speeds given in equation (28), are calculated with the expression

$w_{\text {lim }}=\frac{1}{C \cdot G\left(1-e^{-j \varepsilon}\right)}$

Since $C$ is a real value, real value of $G\left(1-e^{-j \varepsilon}\right)$ is included in the process and the equation becomes as follows,

$$
w_{\text {lim }}=\frac{1}{2 \cdot C \cdot \operatorname{Re}(G(j \omega))}
$$

\section{A COMPARISON OF NUMERICAL CALCULATION AND TEST RESULTS}

In the current study, the workpiece has to AISI-1040 material and $\mathrm{d}=64,9 \mathrm{~mm}$ diameter was attached to a universal machine tool with type TOS SN50C and machined by a tool holder has to dimensions $(b \times h \times l)=(25 \times 25 \times 110) \mathrm{mm}$ with type Kennametal (SDJR$2525 \mathrm{M} 11$ NA3). Data required for calculation, cutting and dynamic parameters determined as a result of model analysis performed by a impact hammer are given in Table 1. Dynamic parameters were determined by using a modal test, CutPro ${ }^{\circledR}$ MalTF 
Table 1. Data of cutting and modal analysis parameters

\begin{tabular}{|c|c|c|c|}
\hline \multicolumn{2}{|c|}{ Cutting Data } & \multicolumn{2}{c|}{ Modal analysis Data } \\
\hline $\mathrm{N}(\mathrm{rpm})$ & 1000 & $\mathrm{C}\left(\mathrm{N} / \mathrm{m}^{2}\right)$ & $1,67 \times 10^{9}$ \\
\hline $\mathrm{s}(\mathrm{mm} / \mathrm{rev})$ & 0,12 & $\mathrm{k}(\mathrm{N} / \mathrm{m})$ & $1 \times 10^{6}$ \\
\hline $\mathrm{w}(\mathrm{mm})$ & 1,2 & $\omega_{\mathrm{n}}(\mathrm{Hz})$ & 773 \\
\hline$\alpha\left(^{\circ}\right)$ & +6 & $\xi(\%)$ & 2 \\
\hline$\beta\left(^{\circ}\right)$ & 70 & $\mathrm{c}(\mathrm{kg} / \mathrm{s})$ & 51,746 \\
\hline
\end{tabular}

software and CutPro ${ }^{\circledR}$ Modal software. The other samples and results related with this subject have given in [20].

A system of orthogonal cutting was dealt with as a SDOF. Analytical modeling of this system and investigation of its stability was conducted in two different forms. The movement of the tool in $(x)$ direction for SDOF system was neglected because the natural frequency of the tool in this direction was very low in relation to the other direction. For this reason, it was accepted that the movement in this direction had no effect on stability limit of orthogonal cutting. Some simplification was made here, too, in order to obtain a useful system model. These were observed to be effective on the results. According to OTF form, which was dealt with first in the study, curves of real and imaginary parts of the transfer function of SDOF system are given in Figure 4. Thus, the frequency corresponding to the minimum negative real part of the transfer function can be predicted as chatter frequency [2]. This value, according to the form calculated here, was predicted to be $789 \mathrm{~Hz}$.

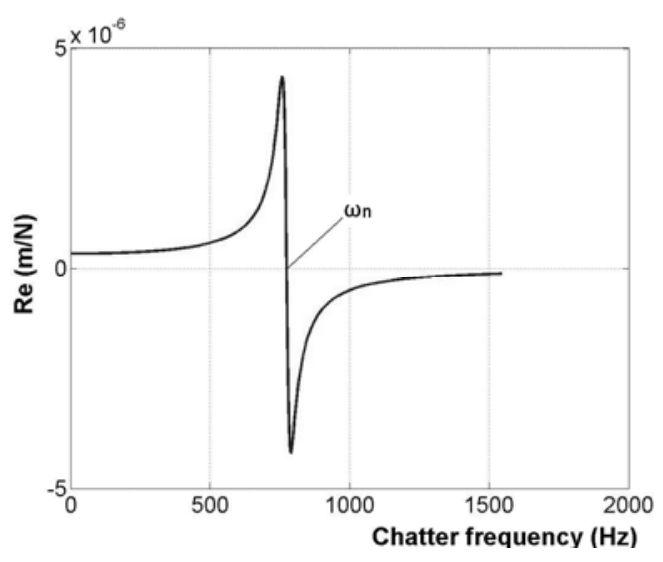

(a)

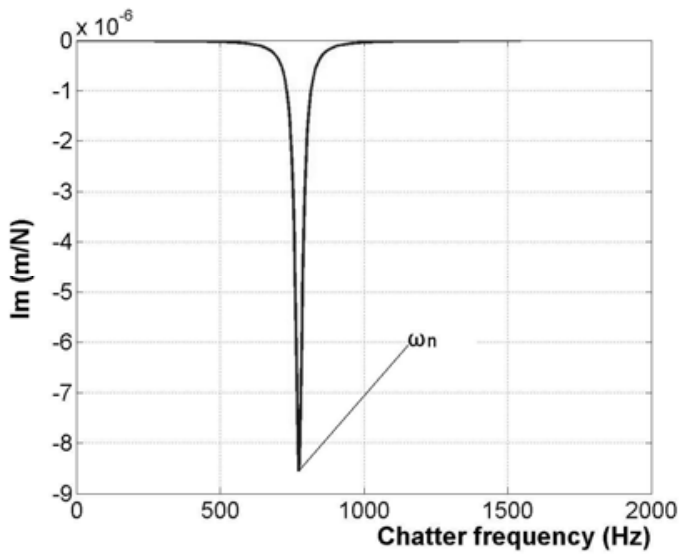

(b)

Figure 4. Transfer function of a SDOF system represented by its (a) real and (b) imaginary parts.

Later, equations of motion of the system were organized in T-decomposition form. For this reason, equations were expressed in terms of relative displacement on the workpiece of the tool ( $l$ ) instead of time. Stability of the system was predicted according to Nyquist criterion. By equation (16), critical orbital curve (unit circle) was 
plotted as $\mathrm{s}=\mathrm{j} . \omega_{\mathrm{i}}, U_{1}(s)=e^{s d \pi}$ and Nyquist place curve was plotted as $U_{2}(s)=\frac{-1}{a_{2} \cdot s^{2}+a_{1} \cdot s+a_{0}}$ as can be seen in Figure $5(\mathrm{a})$. Subscript $(\mathrm{i}=1,2)$ is the number of positive real root of characteristic equation of the system (Equation 17).

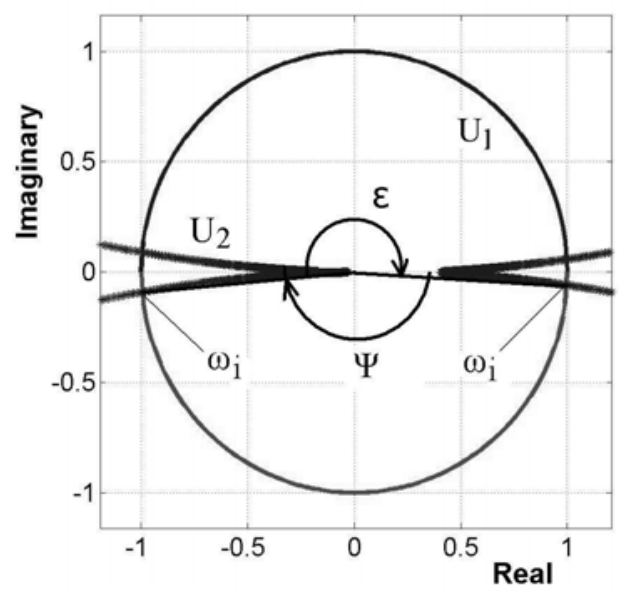

(a)

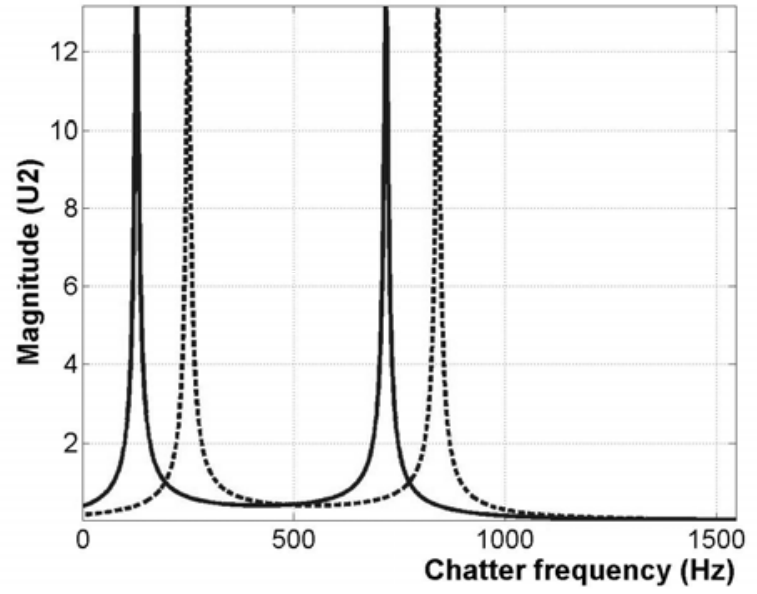

(b)

Figure 5. Plot of (a) $U_{1}$, unit circle and roots in the complex plane (b) magnitude of $\left(U_{2}\right)$

The plot of this curve has been dawn by increasing and decreasing the real roots of characteristic equation of the system (17) in a way that they would enter or leave into the unit circle. System is stable only in intervals where the value of this root is zero. In order to screen the frequency intervals in simulation, the roots were increased in such a way that they would be around natural frequency. Thus, while the curve $U_{2}\left(j \omega_{i}\right)$ enters into the unit circle $U_{1}(j \omega)$ for an increase of one root, the other would leave. The value of frequency at the points where the curve $U_{2}\left(j \omega_{i}\right)$ intersects the unit circle $U_{1}(j \omega)$ is the value of natural frequency $\left(\omega_{n}\right)$. These points are in the regions $3 \mathrm{rd}$ and 4 th of unit circle, respectively. This situation can be observed from magnitude graphics $\left|U_{2}\left(j \omega_{i}\right)\right|$ in Figure 5 (b) plotted for the increase of both root values. Again the frequency value at the point where the two graphics coincide corresponds to the natural frequency of the system. This situation is natural because at this point of coincidence, as can be seen from equation (16) and Figure, is $\left|U_{1}(j \omega)\right|=\left|U_{2}\left(j \omega_{i}\right)\right|=1$. Lines and dashed lines in Figure 5(b) and in all graphics plotted with this method represent respectively the increases related with the curve $U_{2}\left(j \omega_{i}\right)$ for each root value coinciding with $U_{2}$ and the circle $U_{1}$ in the 3 and 4 complex planes. Real and imaginary graphics of the expression $\mathrm{U}_{2}(\mathrm{j} \omega)$ is given in Figure 6. The prediction of chatter in this method was made according to Nyquist criterion [2]. Since the term $C$ in equation (29) was chosen to be real and $\mathrm{w}_{\mathrm{lim}}$ was found to be real, the term $G\left(1-e^{-j \varepsilon}\right)$ should also provide a real value. So the equation in Figure 5(a) should be $\left|U_{2}\left(j \omega_{i}\right)\right|=\left|U_{2}\left(j \omega_{i}\right) e^{-j \varepsilon}\right|$. Thus, $\left(U_{2}\left(\mathrm{j} \omega_{\mathrm{i}}\right)-U_{2}\left(j \omega_{i}\right) e^{-j \varepsilon}\right)$ becomes parallel to real axis and the expression $U_{2}\left(\mathrm{j} \omega_{\mathrm{i}}\right)-U_{2}\left(j \omega_{i}\right) e^{-j \varepsilon}=2 \cdot \operatorname{Re}\left(U_{2}\left(\mathrm{j} \omega_{\mathrm{i}}\right)\right)$ is obtained. According to all said, frequency value corresponding to the point equal to the root value on Nyquist diagram for each imaginary value of the curve $U_{2}\left(j \omega_{i}\right)$ can be 

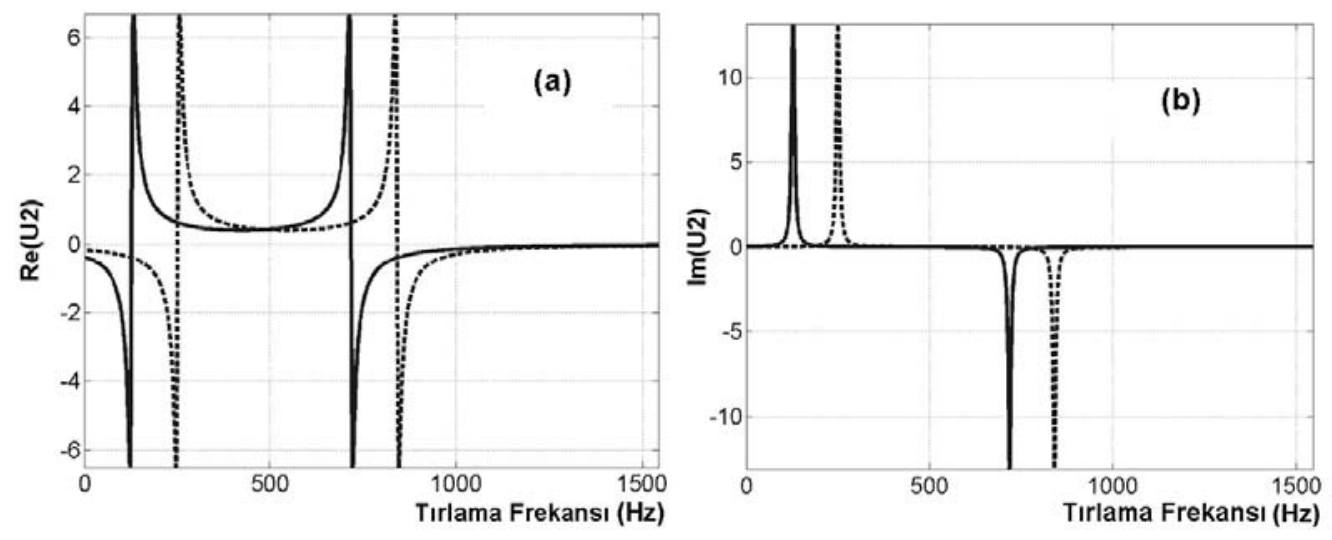

Figure 6. Plot of $U_{2}$, (a) real part and (b) imaginer parts.

taken as the value of chatter frequency (see Figure 6 (b)). According to the calculation form here, this value was predicted to be $779.5 \mathrm{~Hz}$.

SLDs, which were plotted depending on the depth of cut and spindle speed according to the both forms dealt with in the current study, are given in Figure 7. As can be seen in the Figure 7, due to some calculus differences between the two forms, stable depth of cut presents differences at high cutting speeds. This difference between the borders of stable depth of cut gradually narrows at cutting speeds that are appropriate for working in general. This situation is shown in Figure 8. Besides, for spindle speed of $\mathrm{N}=1000 \mathrm{rpm}$, stable depths of cut, according to OTF and T-decomposition forms, were determined as $7,6 \times 10^{-5} \mathrm{~m}$ and $7,3 \times 10^{-5}$, respectively.

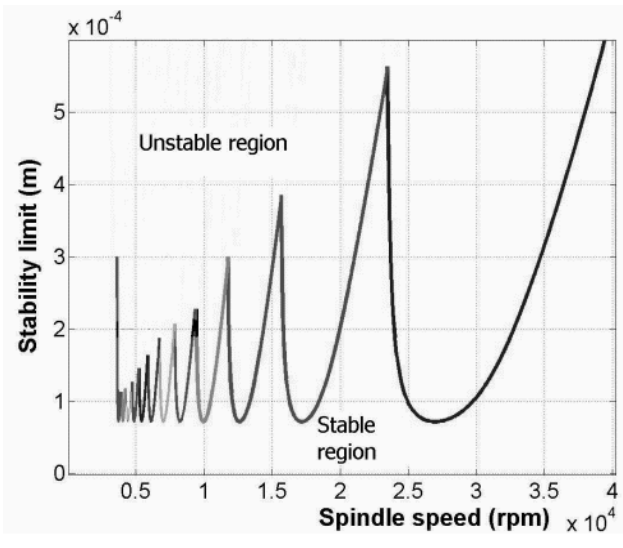

(a)

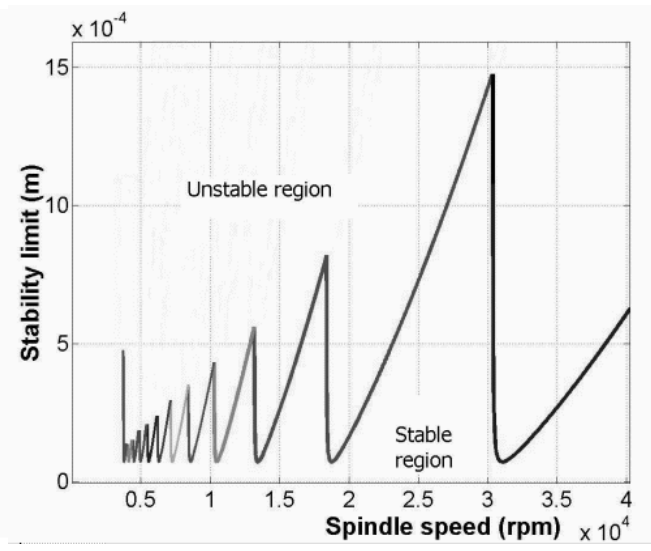

(b)

Figure 7. Plot of SLDs respect to (a) OTF Form and (b) $\tau$ - Decomposition Form. 


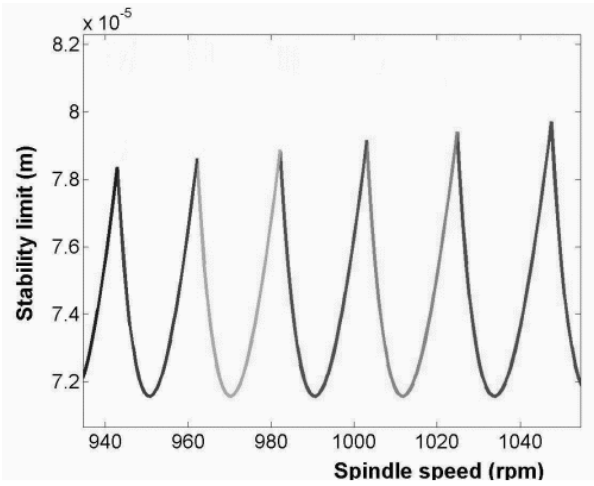

(a)

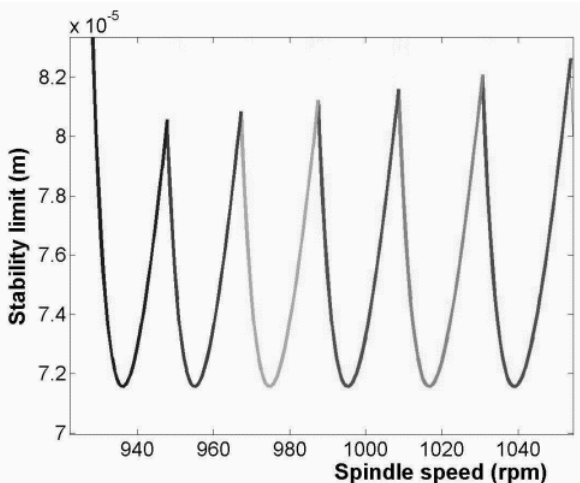

(b)

Figure 8. Plot of SLDs at about $1000 \mathrm{rpm}$ respect to (a) OTF Form and (b) $\tau$ Decomposition Form.

After attaching a accelerometer with a sensitivity of $10,43 \mathrm{mV} / \mathrm{g}$ on the surface of the tool holder in the feed direction, modal analysis test of SDOF were conducted by applying small impacts by impact hammer at appropriate point again in the same direction. Time response of the accelerator was measured and transformed into data frequency domain. For this transformation of time data, Fast Fourier Transform (FFT) was used. At all steps of modal analysis, a portable computer was used for collecting data, calculating modal parameters and presenting the results. All data were collected by using CutPro ${ }^{\circledR}$ MalTF software, and modal analysis was performed using CutPro ${ }^{\circledR}$ MalTF software [1]. Real imaginary graphics of the transfer function of the system obtained from the current study are given in Figure 9 and modal parameter values are given in Table 1.

Cutting test for SDOF system here was performed under the conditions given in Table 1 and without using cooling fluid. Data of cutting test were processed by LabVIEV 7 software that is loaded into the same computer. Noise produced during this process was recorded by a microphone attached to power supply. Noise data recorded was recorded to time domain via LabVIEV 7 software as seen in Figure 10. Then this noise recording was transformed into frequency domain by the software and spectrum graphic was formed. The frequency corresponding to the highest amplitude in the spectrum graphic can be determined as current chatter frequency of the tool performing the process of cutting under these valid conditions. The chatter frequency determined here is $763 \mathrm{~Hz}$.

\section{RESULTS}

The current study focused on a turning system with SDOF in orthogonal cutting process. Prediction of the chatter frequency of the system and the investigation of its stability was conducted in two separate forms; one in the form oriented transfer function (OTF), and the other in the form of applying T-decomposition form to Nyquist criterion. Results obtained from both forms were compared with the modal analysis conducted and the results of cutting test. Prediction chatter frequency in both calculation forms explained in this study, as expected [1,2], is greater than the natural frequency that was found as a result of the modal analysis of the system. But according to the result of this cutting test, it was found that the value of this frequency is, in fact, under 

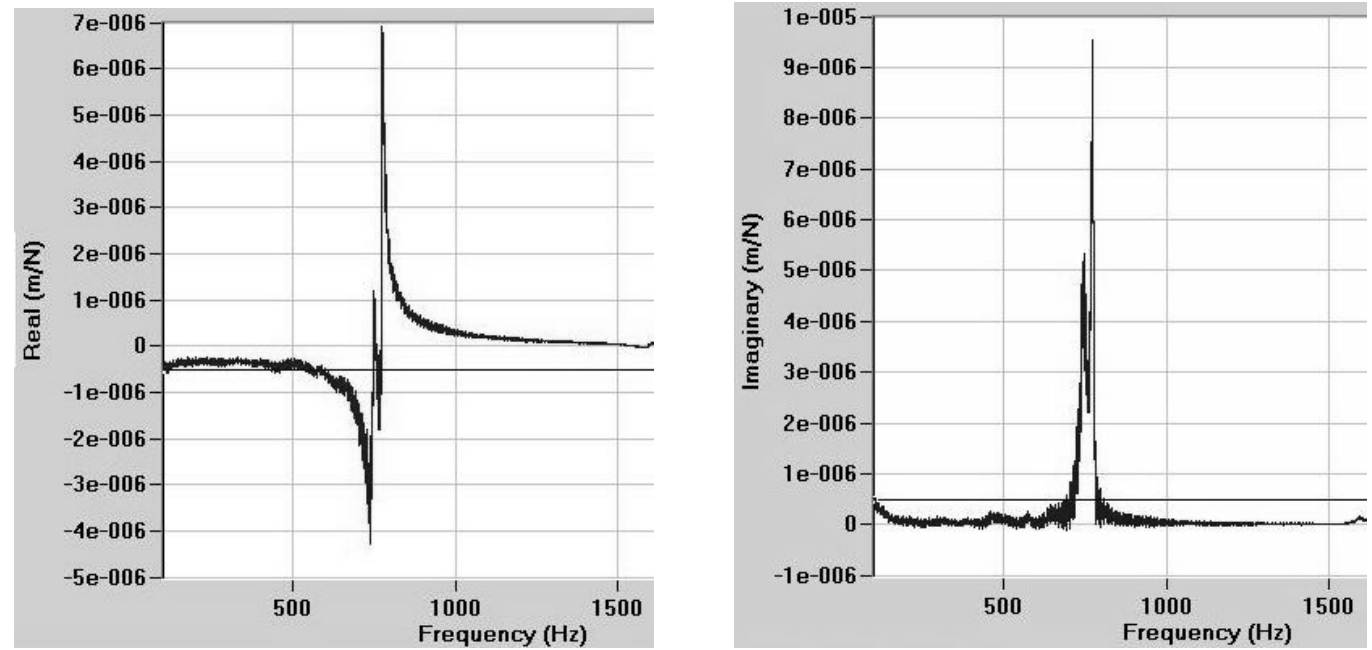

Figure 9. Real and Imaginary graphics obtained by modal analysis of the SDOF system.
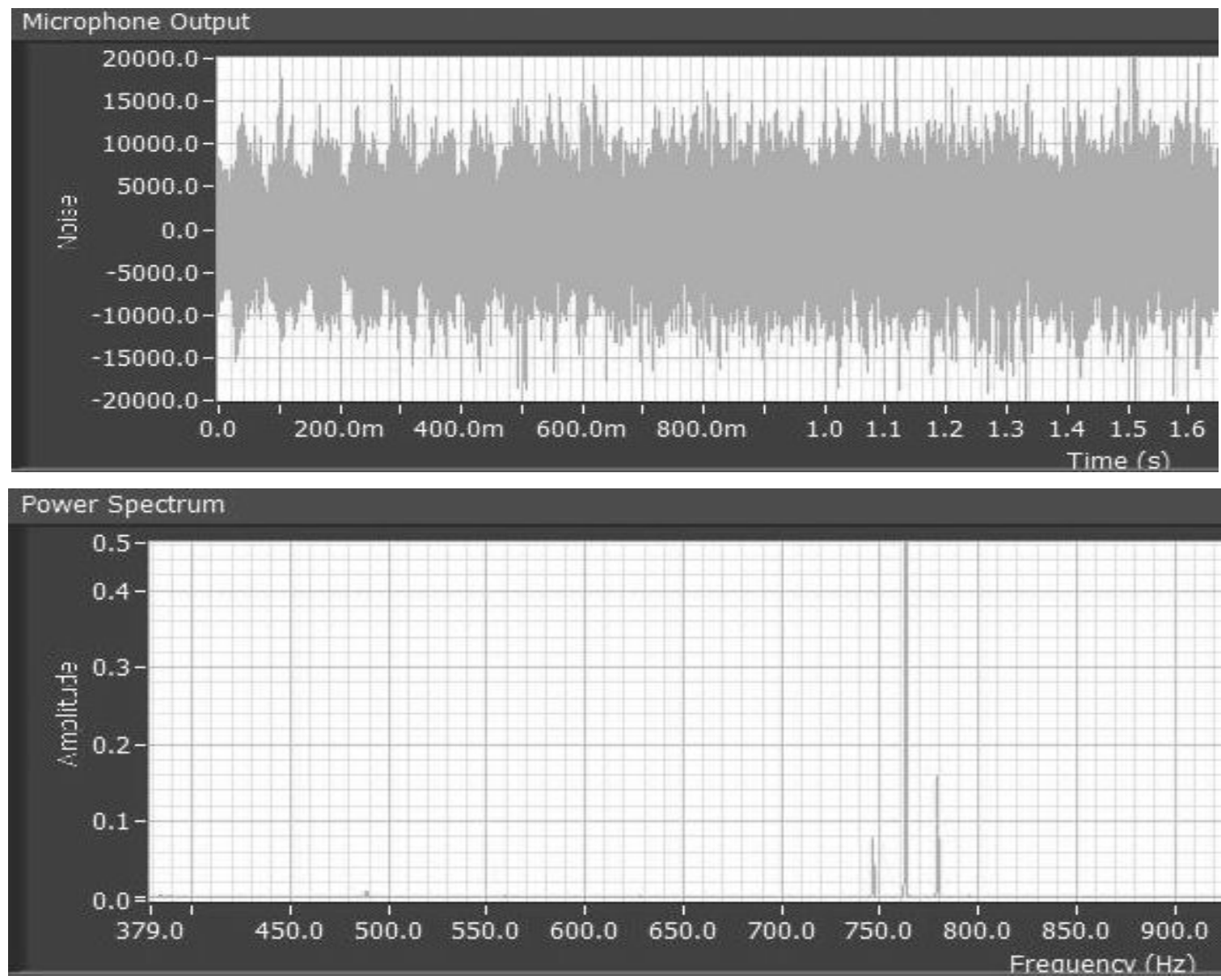

Figure 10. Graphics of Microphone output and Power spectrum for the SDOF system. 
the natural frequency of the system. The fact that both of the calculation forms have analytical and linear structure may be the factor that affects these results. Besides, in predicting chatter frequency, the clamping situation of the tool holder is fairly important. During various modal analysis made, it was observed that a second mode appeared in real and imaginary graphics when the attachment of the tool holder was not rigid enough. Another factor affecting these results is that the damping in process and the damping in cutting process were not taken into account. Stability of the system is affected by dynamic factors such as nose radius of the tool holder, sharpness or bluntness and built-up edge. Although it is present in literature how these factors affect to the stability of the system, there doesn't still exist any complicated modeling that takes into consideration all of these dynamic factors. Although all these effects were considered in this current study, a deviation of $3.3 \%$ in OTF form and $2.12 \%$ deviation in T-decomposition form was observed in chatter frequency prediction compared with current chatter frequency. Under current working conditions, according to both forms of calculus, the results in the prediction of the stability of the system, results were quite similar. However, it was observed that this difference is larger in high cutting speeds. The other samples and results related with this subject have given in [20].

\section{REFERENCES}

[1]. Altintas, Y., Manufacturing Automation; Metal Cutting Mechanics, Machine Tool Vibrations and CNC Design, Cambridge University Press, 2000.

[2]. Tlusty, G., Manufacturing Processes and Equipment, Prentice Hall, New Jersey, 2000.

[3]. Leigh, E., Tlusty, J., Schueller, J., Applying High-Speed Machining Techniques on Rotor Grip Configurations, American Helicopter Society 55th Annual Forum, Canada, 25-27 May, 1999.

[4]. Badrawy, S., Cutting Dynamics of High Speed Machining, Wolf Tracks Vol. 8, Issue 1, 2001.

[5]. Davies, M.A., Burns, T.J., Schmitz, T.L., High-Speed Machining Processes: Dynamics on Multiple Scales, pp. 7-19. Dynamics and Control of Mechanical Processing Proceedings of the Workshop Organized by Working Group 2 "Nonlinear Dynamics and Control" in COST Action P4, Budapest, Technical University of Budapest (1999)

[6]. Koelsch, J.,R., High-Speed Machining: A Strategic Weapon, Machine Shop Guide Web Archive-November 2001.

[7]. Taşkesen, A., Ercan, Y., Theoretical Analysis And Prediction Of Machine Tool Stability And Chatter Vibration During Orthogonal Metal Cutting With A One Degree Of Freedom Model, 11. National Machine Theory Symposium, Gazi University, Ankara, 4-6 September 2003.

[8]. Landers, R.G., Ulsoy, A.G., Chatter Analysis of Machining Systems With Nonlinear Force Processes, ASME International Mechanical Engineering Congress and Exposition, Atlanta, Georgia, November 17-22, DSC Vol. 58, 183190 pp., 1996.

[9]. Nosyreva, E.P., Molinari, A., Nonlinear Analysis of Chatter in Orthogonal Cutting, Dynamics of rigid or flexible systems, C.R. Acad. Sci. t. 325, Serie II b, p. 435442, Paris, 1997.

[10]. Landers, R.G., Lu, Y-W., Stability Analysis of Nonlinear Machining Force Controllers, American Control Conference, San Diego, California, 678-683pp, June 2-4, 1999. 
[11]. Xiao, M., Karube, S., at all, Analysis of chatter suppression in vibration cutting, International Journal of Machine Tools \& Manufacture vol. 42, 1677-1685pp., 2002.

[12]. O'Reilly, T., The Effect of Variable Delay Length on Stability in Orthogonal Cutting, Machine Dynamics Research Lab.,Master of Science, The Pennsylvania State University, Mechanical Engineering, May 1998, 142 pp.

[13]. Lagö, T.L., Sven Olsson, S., at all., Performance of A Chatter Control System for Turning and Boring Applications, 4 th GRACM Congress on Computational Mechanics, Patras, 27-29 June, 2002.

[14]. Altintas, Y. and Budak, E., Analytical Prediction of Stability Lobes in Milling, Annals of the CIRP, Vol. 44/1, 357-362pp., 1995.

[15]. Olgac, N., Hosek, M., A New Perspective and Analysis for Regenerative Machine Tool Chatter, International Journal of Machine Tools \& Manufacture, Vol. 38, 783-798 pp., 1998.

[16]. Budak, E., An Analytical Design Method for Milling Cutters With Nonconstant Pitch to Increase Stability, Part I: Theory, Journal of Manufacturing Science and Engineering, Transactions of the ASME Vol. 125, 29-34pp, 2003.

[17]. Budak, E., An Analytical Design Method for Milling Cutters With Nonconstant Pitch to Increase Stability, Part II: Application, Journal of Manufacturing Science and Engineering, Transactions of the ASME Vol. 125, 35-38pp, 2003.

[18]. Pan, J., Su, C-Y., Chatter Suppression With Adaptive Control in Turning Metal Via Application of Piezoactuator, Proceedings of The 40th IEEE Conference on Decision and Control, Orlando, Florida USA, December 2001.

[19]. Dohner, J.L., Lauffer, J.P., at all., Mitigation of Chatter Instabilities in Milling by Active Structural Control, Journal of Sound and Vibration, 2003.

[20]. Türkeş, E., Theoretical and Experimental Analysis of Process Damping in Machine Tool Chatter Vibration, Doctoral Dissertation, Eskişehir Osmangazi University, Applied Science Institude, 2007. 